\title{
Program evaluation of Sea Mar's Chronic Care Program for Latino and Caucasian patients with type 2 diabetes: providers and staff perspectives
}

This article was published in the following Dove Press journal: Journal of Multidisciplinary Healthcare

26 September 2012

Number of times this article has been viewed

\author{
Gail E Bond' \\ Laurie Rechholtz' \\ Christina Bosa' \\ Celine Impert ${ }^{1,2}$ \\ Sara Barker ${ }^{2}$ \\ 'College of Nursing, Seattle \\ University, Seattle WA, USA; ${ }^{2}$ Sea Mar \\ Community Health Center, Seattle, \\ WA, USA
}

Problem statement: Unprecedented consumption of health care resources in the USA coupled with increasing rates of chronic disease has fueled pursuit of improved models of health care delivery. The Chronic Care Model provides an organizational framework for chronic care management and practice improvement. Sea Mar, a community health care organization in Washington state, implemented the Chronic Care Model, but has not evaluated the outcomes related to provider and staff satisfaction. The specific aim of this project was to evaluate the effectiveness of the Chronic Care Model with the addition of the Chronic Care Coordinator role.

Approach: A descriptive method was used, which incorporated quantitative, and qualitative data from providers and clinic staff collected through a Web-based survey consisting of Likerttype questions sent via an electronic link.

Results: This evaluation identified the strengths of and barriers to the chronic care model with a focus on provider and staff satisfaction regarding patient care since the addition of the Chronic Care Coordinator role. We found a high appreciation (94\%) and acceptance of the role; $80 \%$ agreed that the Chronic Care Coordinator was well-integrated into clinic operations. Major strengths of the program included more patient education, better follow-up, and improved team communications. Barriers to success included limited provider access, confusion regarding role expectations of the Chronic Care Coordinator, inconsistent communications, and Chronic Care Coordinator turnover.

Conclusions/recommendations: Our findings help to validate the importance of community health organizations such as Sea Mar, the utility of the chronic care model, and the potential value for specific roles such as the Chronic Care Coordinator to positively impact quality of care by helping to empower patients to improve self-management and ultimately impact patient outcomes. However, future studies involving larger samples are needed to further explore themes among staff and patients.

Keywords: case manager, chronic care model, program evaluation, type 2 diabetes, Care Coordinator

\section{Background}

Turmoil in the United States (US) health care system threatens the delivery of highquality patient care, and the Institute of Medicine $^{1}$ has made coordination of care a national priority for improving health care. The Institute of Medicine established a call to action to develop organizational models to improve access, delivery, and quality of care for patients with chronic health problems. As of 2009, 133 million, or nearly half of all Americans, had at least one chronic illness, and half of those had multiple chronic diseases. ${ }^{2}$ In 2005, 63 million people had multiple chronic illnesses, and that number is expected to reach 157 million in $2020 .{ }^{3,4}$ Healthy People 2020 continues to
Correspondence: Gail E Bond Seattle University, College of Nursing, 90I 12 Ave, Seattle, WA 98122-1090, USA

Fax +I 2062965544

Email bondg@seattleu.edu 
emphasize the need for improved models of health care delivery to reduce disparities in care. ${ }^{5}$

According to the Centers for Disease Control and Prevention, 25.8 million people in the US ( $8.3 \%$ of the population) suffer from diabetes, with 18.8 million diagnosed and 7.0 million undiagnosed. More than $90 \%$ of those have been diagnosed with type 2 diabetes mellitus. ${ }^{6}$ By 2035, this number is anticipated to increase by more than $200 \%$ to nearly 64 million. ${ }^{7}$ Type 2 diabetes represents a significant burden on health care resources and is a leading cause of suffering. ${ }^{8}$ Diabetes disproportionately affects under-served and specific ethnic and minority populations; $9.8 \%$ of non-Hispanic whites, $7.5 \%$ of Asian Americans, $10.4 \%$ of Hispanics, and $14.7 \%$ of non-Hispanic blacks have been diagnosed with diabetes as of 2007. Among Hispanics, rates were 8.2\% for Cubans, $11.9 \%$ for Mexican Americans, and $12.6 \%$ for Puerto Ricans. ${ }^{9}$ Significant complications are associated with the disease, including heart disease, stroke, hypertension, kidney disease, retinopathy, blindness, nervous system disease, and lower-limb amputations. ${ }^{6,8}$ These complications have resulted in people with diabetes averaging medical expenses that are 2.3 times higher than if they did not have diabetes. ${ }^{6,8}$ As the incidence of diabetes mellitus reaches epidemic proportions while continuing to incite upward spiraling costs, it becomes increasingly imperative to implement more effective models of health care delivery to help reduce the complications associated with this disease.

One such model is the Chronic Care Model (CCM), developed by Wagner et al at the MacColl Institute of Health. ${ }^{10}$ Wagner and colleagues researched best practices for the care of people with chronic illness, noting the importance of the ongoing interaction between patients and their health care providers, and designed the $\mathrm{CCM}$, an organizational and conceptual framework to improve the management of chronic illness. ${ }^{11}$ This model offers hope for the effective and efficient transformation of health care systems. ${ }^{12-14}$ Additional research has provided evidence of the CCM's capacity to reduce the economic burden associated with diabetes and chronic care management. ${ }^{10,15}$ The CCM has become a well-embraced organizational construct to help guide chronic care improvement and care coordination. ${ }^{16}$ The model provides a multidimensional solution for the complex problem of diabetes care and encourages physicians and health care teams to organize their practices to achieve high quality care. ${ }^{12,13}$ Since the inception of the CCM, numerous health care organizations have employed one or more components to improve the care of people with chronic disease. ${ }^{17}$ Others note that while the CCM can help organizations improve quality and satisfaction with care, the model requires further customization and revision relevant to individual clinical settings. ${ }^{18}$ The model acknowledges six primary domains which have been leveraged to varying degrees by health care organizations and updated in 2004 with subthemes: ${ }^{3,12}$

1. community resources (subtheme: policies)

2. health system (subthemes: care coordination and patient safety)

3. self-management support (subthemes: help motivate patients and teach self-management skills)

4. delivery system design (subthemes: case management and cultural competency)

5. decision support (subthemes: evidence-based treatment guidelines)

6. clinical information systems to remind staff and providers of evidence-based best practices, and easily accessible data on patient and provider performance on key metrics (subtheme: care coordination, especially using an electronic health record to enable development and management of "registries" of patients that help plan individual and population-based care).

Sea Mar (Seattle, WA) provides an example of a community health organization customizing the CCM to improve care delivery to low-income, underserved, and uninsured patients suffering from diabetes and other chronic illnesses. Type 2 diabetes is the second most common chronic disease amongst Sea Mar patients. Since 2000, Sea Mar has been working to improve patient and staff interactions and to enhance quality measures based on the CCM. Sea Mar's network of health care clinics primarily serves low-income Hispanic (42\%) and non-Hispanic Caucasian (43\%) patients. Sea Mar introduced the CCM with the implementation of five CCM domains (health system, self-management support, decision support, delivery system design, and clinical information system) in ten of their primary care clinics over the past decade. See Figure 1 for a graphic illustration of the CCM domains. In 2008, consistent with the evolved CCM, Sea Mar augmented their delivery system with the implementation of the Chronic Care Coordinator (CCC) role. The objective was to help providers and staff better coordinate care to meet the needs of chronically ill patients, primarily through patient education, supporting practice guidelines, self-management coaching, and improved interactions between the health care team and patients.

Sea Mar identified the need for a program evaluation after on the implementation of the $\mathrm{CCC}$ role. This evaluation examined Sea Mar's CCC implementation, structure, and function based on perceptions, attitudes, and experiences 


\section{Chronic care coordinator}

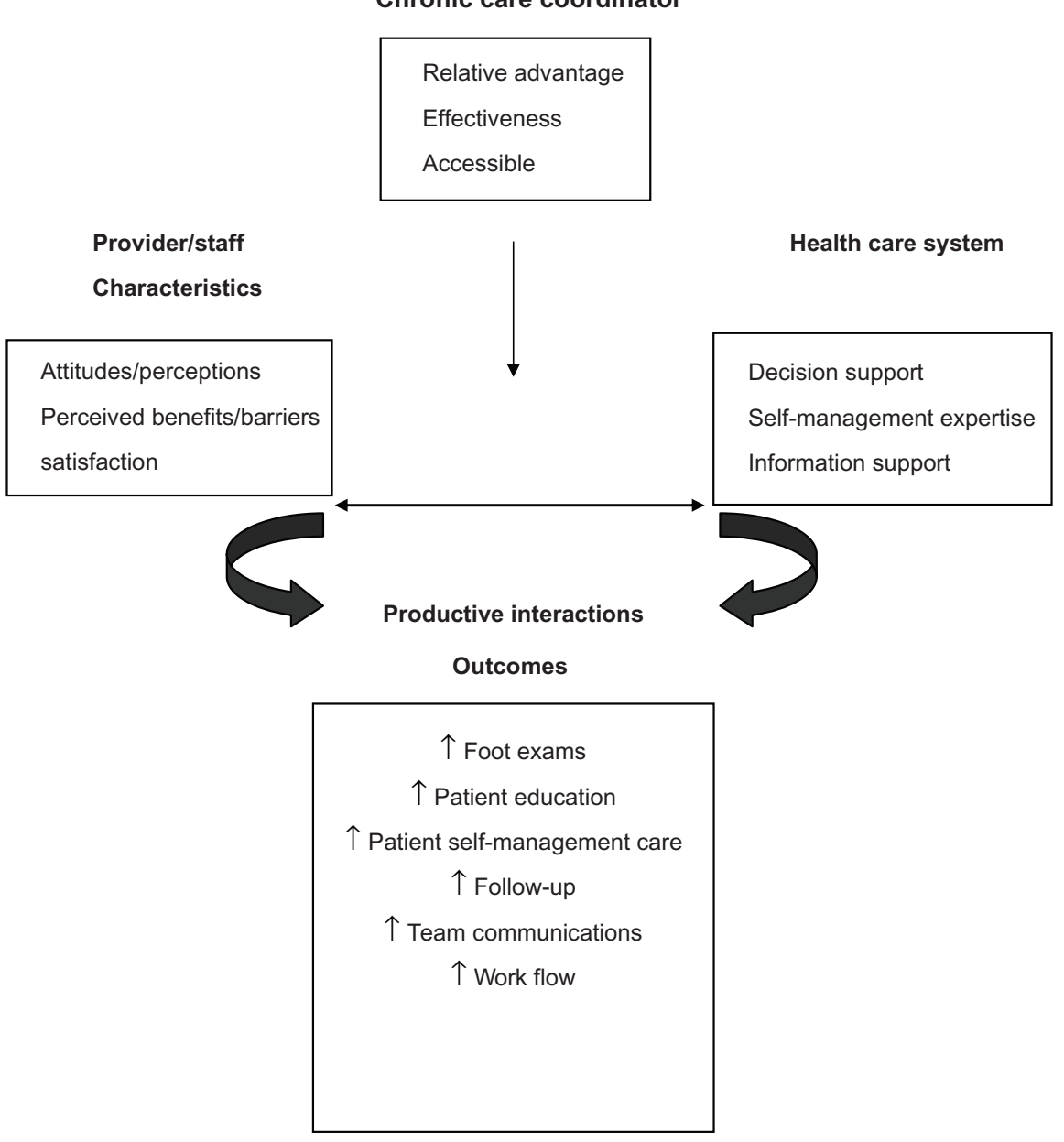

Figure I Integration of the Chronic Care Coordinator into the chronic care model for effective diabetes care.

of providers and staff. The purpose of this evaluation of the $\mathrm{CCM}$ was to assess provider and medical staff perceptions and attitudes regarding whether the model had improved the care of patients with diabetes at Sea Mar and how the model could be improved. The evaluation assessed relevance, acceptability, and satisfaction with the care coordinator, as well as the barriers to adoption from the perspective of the providers and clinical staff. This project collected data that explored the fidelity of the implementation of the care coordinator role, as well as the program strengths and weaknesses, through the experiences and perspectives of chronic care coordinators and patients. The findings from the patients and care coordinator interviews are not reported in this paper. The research questions asked regarding provider perceptions included:

1. Is there general awareness and acceptance of the CCC role among providers and staff?

2. Do providers and clinical staff believe the quality of care delivered to patients using the CCC role has improved compared to prior years?
3. Does the care team feel their effectiveness or efficiency as care providers has improved with the addition of a CCC role to the team?

4. Is there a perception of improved patient outcomes since the addition of the CCCs?

5. What are provider and staff attitudes and perceptions about the benefits and barriers to the implementation of the $\mathrm{CCC}$ role?

\section{Materials and methods Research design}

This evaluation used a descriptive mixed methods approach. Qualitative and quantitative data were collected from a Web-based online survey. This evaluation was a joint collaboration between Seattle University and Sea Mar and was approved by the Institutional Review Board at Sea Mar and Seattle University. Evaluation research is a systematic, objective process to determine the impact, effectiveness, and importance of the changes implemented 
in an organization and how well patients have adapted to the changes. ${ }^{19}$

\section{Study sample}

Primary care providers (PCP), as well as other members of the care team, were asked to complete the survey to better understand whether the model was working as intended (employees feel empowered and supported to ensure that patients are getting the right care) and how it could be further strengthened. Inclusion criteria were (1) respondents were employed by Sea Mar and (2) serving patients with type 2 diabetes.

\section{Survey development}

The survey developed for this study, the Staff and Provider Attitudes and Perceptions Survey, consisted primarily of questions using a 5-point Likert-type rating scale ranging from strongly disagree to strongly agree. This study survey also included open-ended, multiple-choice, true/false and yes/no questions and was guided by the following broad research question: What are provider and clinical staff attitudes and perceptions regarding the benefits and barriers to the CCC implementation and ongoing role within Sea Mar's CCM? There were 26 questions regarding how care is delivered to diabetes patients at Sea Mar versus how care was delivered prior to the implementation of CCCs. The survey was similar to the Assessment of Chronic Illness Care (ACIC) survey $^{20}$ in that both recognized the six core domains of the CCM: organization of delivery system, community linkage, self-management support, decision support, delivery system design, and clinical information system. The ACIC survey was designed to be taken by providers and others to help organizations evaluate the strengths and weaknesses of their delivery of care for chronic illness and to evaluate improvements made as a result of interventions. ${ }^{20}$ See Table 1 for a sample listing of survey questions.

\section{Study procedures}

Clinic managers recruited providers and staff via email sent to the employee email inbox. The email included a short note explaining the purpose of the survey and, if interested, the employee could click a link taking them to the study survey. All participants provided consent to continue to the survey questions. A reminder email was sent again 2 to 3 weeks later. The survey solicited perceptions and attitudes regarding how care was delivered to patients with diabetes at Sea Mar versus how it was delivered prior to the implementation of CCCs.
Table I Sample of questions on the staff and provider attitudes and perceptions survey (SPAPS)

I. Workflow at Sea Mar, around the care of patients with diabetes, is smooth and efficient.

2. Workflow at Sea Mar, around the care of patients with diabetes, has improved in the last 2 years.

3. The Chronic Care Coordinator makes a positive difference in clinical work flow, especially in treating patients with diabetes.

4. The quality of care I provide our patients with type 2 diabetes has improved over the last couple of years.

5. I believe outcomes of Sea Mar patients with diabetes have improved over the last couple of years.

6. Our Chronic Care Coordinator has helped to improve the care of my patients with type 2 diabetes.

7. I see more patients with type 2 diabetes than 2 years ago.

8. The Chronic Care Coordinator role is well-integrated at my clinic.

Note: Responses to questions were based on a 5-point Likert-type rating scale ranging from strongly disagree to strongly agree.

To test the survey and estimate the length of time needed to complete the survey, volunteer staff, a Sea Mar leadership team, Group Health Research Institute leadership, nursing faculty, and nurses took the survey. Each person reported taking approximately 3 to 5 minutes to complete the survey. Additionally, the survey was pilot-tested in a clinic excluded from primary data collection. Data from the pilot survey was used to improve flow and comprehension of questions. Surveys were sent via email by clinic managers at nine clinics to the following providers: physicians, nurse practitioners, physician assistants, medical assistants, and other staff.

\section{Data analysis}

Data analysis was guided by our research questions. The survey data were analyzed using Survey SPSS (IBM, Armonk, NY) and Excel (Microsoft, Redmond, WA) spreadsheets. Descriptive statistics were used to describe the sample and response rates to survey questions. Comments were evaluated for qualitative content by organizing them into the areas of structure, process, and outcomes and searching for themes and patterns of repetition using content analysis.

\section{Results}

The intent of the project was to identify provider and staff perceptions and attitudes about the benefits and barriers to the implementation of the CCC role at Sea Mar and whether it was helpful for improving patient care. Findings were compared and contrasted between providers and staff as well as those employed at Sea Mar for more than 2 years versus those employed for a shorter time period. The survey generated 96 respondents with a $75 \%(\mathrm{n}=72)$ completion rate. Providers $(n=22$ or $31 \%)$ included 17 physicians, 
four nurse practitioners, and one physician assistant. Staff ( $n=50$ or $69 \%$ ) included nurses, medical assistants, lab staff, health educators, clinic managers, and support personnel. Physicians and medical assistants represented the largest response groups of $24 \%$ and $22 \%$, respectively. Clinic participation rates ranged from $6 \%$ to $21 \%$. Among respondents who completed the survey, $81 \%(\mathrm{n}=58)$ had been employed for 1 year or more and $65 \%(n=47)$ had been employed for more than 2 years.

There was high general awareness of the CCC with $94 \%$ of those surveyed claiming to know the name of their CCC and $96 \%$ of those correctly citing the name. Overall, the CCC was regarded as part of the team, and even more so than the physicians. When asked who comprised the chronic care team, $94 \%$ of participants recognized the CCC, $90 \%$ cited physicians, and $88 \%$ cited medical assistants. Other team members commonly cited included the health educator $(87 \%)$ and lab personnel (83\%). CCCs were also wellintegrated into their respective teams according to $81 \%$ (58) of respondents. However, attendance at monthly chronic care meetings was 39\% overall, and these meetings were better attended by staff (64\%) than by providers (23\%). Only one provider per team attended; thus, some providers would not be in attendance.

Among all providers and staff surveyed, there was a perception of improved quality of patient care; $67 \%$ agreed or strongly agreed that that the quality of care they provided patients with type 2 diabetes had improved over the last couple of years and $80 \%$ agreed or strongly agreed that the CCC helped them do this.

Providers and staff who had worked at Sea Mar for more than 2 years perceived that the general care of patients with type 2 diabetes improved compared to care prior to the implementation of the CCC. A total of $92 \%$ agreed or strongly agreed that care provided for patients with type 2 diabetes had improved compared to 2 years ago.

Sea Mar management wished to ensure that not only was the care model helping to improve care for patients with diabetes, but also that the care model did so without interrupting work flow. Those surveyed indicated their effectiveness and efficiency had improved with the addition of a care coordinator to their team. In general, providers and staff $(69 \%$; $\mathrm{n}=50$ ) reported improved workflow since implementation of the CCC 2 years ago.

When asked whether workflow was currently smooth and efficient, 63\% $(\mathrm{n}=45)$ agreed or strongly agreed, suggesting that despite progress, opportunity still exists for improvement. CCCs have been credited with helping to improve work flow by $78 \%(\mathrm{n}=56)$ of respondents; $67 \%(\mathrm{n}=48)$ also acknowledged the contribution of team collaboration in improving workflow. Eighty-three percent of those surveyed $(n=60)$ agreed or strongly agreed that the CCC made a positive difference in clinical work flow, especially in treating patients with diabetes. Additionally, the CCC was credited with helping to improve the efficiency of care for patients with diabetes according to $82 \%$ of providers $(n=18)$ and $56 \%(n=28)$ of staff.

Eighty percent of those employed for more than 2 years believed that outcomes for Sea Mar patients with diabetes, especially process outcomes, have improved over the last couple of years. This perception was not as strong among the entire group surveyed (74\%), and particularly not among the provider-only group. Only $48 \%$ either agreed or strongly agreed with the statement. However, all indicated multiple areas of improvement over the past 2 years. The most commonly cited improvements were access to a comprehensive health care team $(58 \%)$, more patients receiving annual eye and foot exams (51\% and 61\%), and staff perceptions that glucose management had improved (52\%). Only 23\% of providers felt that patients had improved in the overall management of their diabetes.

Those surveyed reported the greatest benefits to patients over the last 2 years included better education (70\%), faster completion of lab work (64\%), and attaining a better understanding of how to manage diabetes $(64 \%)$. The data supporting these outcomes were even stronger among those employed at Sea Mar for more than 2 years at $81 \%, 77 \%$, and $72 \%$, respectively. There were differences, however, between the groups (provider versus staff) in terms of the benefits provided by the CCC role. Eighty-six percent felt that improved team communications and having someone to confide in $(77 \%)$ were the most important benefits. Staff and those employed for more than 2 years indicated that better access to resources and referrals ( $82 \%$ and $75 \%$, respectively) and having a better plan for managing patient care $(78 \%$ and $75 \%$ ) were more important benefits. See Table 2 for the primary barriers to adoption of the $\mathrm{CCC}$ role identified from the provider and staff comments.

In summary, the major strengths of the program included providing more patient education, better follow-up, ensuring patients obtain needed labs, and improved team communications. Barriers to its success included limited provider access, confusion regarding expectations of the $\mathrm{CCC}$, a new electronic health record (EHR), and inconsistent communications and patient follow-up. Additionally, CCC knowledge gaps and turnover tend to impede care delivery. 
Table 2 Primary barriers to adoption of the Chronic Care Coordinator (CCC) role identified from the provider and staff comments

\section{Structure}

I. Limited provider access.

2. Confusion regarding role expectations of the CCC.

3. Cumbersome Electronic Health Record (EHR) not yet adapted to chronic care follow-up.

Process

4. Inconsistent team communications.

5. Inconsistent follow-up.

Outcomes

6. CCC disease knowledge $\rightarrow$ patient education $\rightarrow$ patient activation.

7. Reduced CCC turnover rate.

\section{Discussion}

The purpose of this evaluation of the CCM was to assess provider and medical staff perceptions and attitudes of whether the model had improved the care of patients with diabetes at Sea Mar and how the model could be improved. We believe our findings enhanced the existing body of literature on the CCM about provider and staff perceptions regarding the impact of care coordinators on the quality of patient care. Relevance, acceptability, and satisfaction with the care model, as well as barriers to adoption, were assessed by providers and staff. Results showed positive support for continuing with the CCM at Sea Mar and for maintaining the CCC role for the care of patients with diabetes.

Those surveyed provided answers to the questions we set out to answer in this research evaluation. These questions included: (1) Is there general awareness and acceptance of the CCC among providers and staff? (2) Do providers and clinical staff believe the quality of care delivered to patients using the CCC role has improved compared to prior years? (3) Does the care team feel their effectiveness or efficiency as care providers has improved with the addition of a care coordinator to the team? (4) Is there a perception of improved patient outcomes since the addition of care coordinators? (5) What are provider and staff attitudes and perceptions about the benefits and barriers to the implementation of the CCC role?

The CCM was highly relevant and well-accepted by providers and staff. Specifically, providers and clinical staff were aware of the CCC's role and have embraced it as part of the team (research question number one). Preliminary data analysis from our patient and care coordinator interviews provide validation from the provider and staff on how well the care coordinator role has been accepted.

We found that providers and clinical staff believed the quality of care delivered to patients using the Care
Coordinator role had improved compared to prior years (research question two). Additionally, the care team felt their effectiveness and efficiency as care providers had improved with the addition of a care coordinator (research question three) and they believed patient outcomes had improved since the addition of the CCC (research question four). Sea Mar also gained greater insight into key provider and staff attitudes and perceptions about the benefits and barriers to the implementation of the $\mathrm{CCC}$ role (research question five).

Quality of care and improved patient outcomes span a broad spectrum ranging from process and process outcomes, such as getting annual retinal and foot exams, monitoring blood glucose and blood pressure levels, and routine follow-up visits, to health metrics, such as lower levels of blood glucose, blood pressure, cholesterol, vascular disease, and end organ damage. There are myriad dimensions of quality of care and patient outcomes, so we recommended reframing these questions or goals to be more focused and actionable for Sea Mar in both the short and long term. Ideally, these will be goals that are specific, measurable, achievable, realistic, time-specific, and identified by the patient, not just an American Diabetes Association (ADA) guideline. Finally, providers and staff reported varying degrees of perceived benefits and barriers to success. It is possible that providers and staff differ in their expectations of quality of care, patient outcomes, and the ways in which care coordinators can be best integrated into the care team. Further analysis of the survey data, supplemented with interviews or focus groups, could generate content to validate these findings and perhaps reveal more granular findings and implications. Future surveys can also be tailored to key groups such as providers and medical assistants.

In summary, there is a wealth of literature endorsing the CCM and little information regarding provider and staff attitudes and satisfaction with the CCM. Existing research captures provider adherence to ADA protocol but not the provider's perception about delivering care to chronically ill people via the CCM. This study adds new information to the literature regarding provider and staff attitudes and perceptions about both the benefits of and barriers to the CCM through the implementation of the CCC role.

\section{Our recommendations for Sea Mar consideration}

The health of an organization is dependent on the satisfaction of employees. Additionally, due to concerns regarding reprisal, some employees may not be willing to overtly voice concerns or discontent until organizational damage has been done. Therefore, it is important to consider the perspectives 
of all employees who participate in the care of people with chronic illness by giving them a forum to express these concerns and to do so with anonymity if desired. The research team encouraged Sea Mar to routinely survey providers and staff regarding their perceptions and ideas for improvement. This study can be used as a starting point for developing baseline assessments of general attitudes regarding patient care.

We believe this model can be improved upon with persistent yet incremental modification. The following are our recommendations for beginning to build on existing strengths and address major areas for improvement, organized by the themes outlined below.

1. Identify patients who have difficulty in their selfmanagement care and redirect them to other appropriate resources, if possible.

2. Clarify expectations of CCC (knowledge and performance).

3. Provide EHR training specific to diabetes care.

4. Improve team communications.

5. Increase patient follow-up by creating a system through the EHR that ensures patients obtain labs that they need.

6. Improve CCC knowledge and skills through training and education to improve team efficiency and effectiveness.

7. Conduct a root cause analyses to determine underlying cause for the CCC turnover rate.

\section{Limitations}

These findings were limited to a small sample of 22 providers and 50 staff. There were no baseline data for comparison, so this study was, in part, retrospective in nature. Ideally, there would be a baseline survey and then periodic follow-up surveys. Participants were recruited by electronic mail from one health care institution, Sea Mar Community Health Center, and may not be representative of providers and staff in other geographic venues or among those who serve a more affluent patient population. While the findings of this study may not be considered generalizable to all clinic employees, the data provide a good representation of provider and staff perspectives at the various Sea Mar medical clinics. These findings would be strengthened if patient outcomes had been collected to validate the implementation of the CCM.

There was the possibility of several types of bias, including selection, central tendency, social desirability, and acquiescence. Some of this cannot be avoided because people interested in the CCM may be more inclined to take the survey, participants may have avoided extreme response categories for fear that the survey was not really anonymous, or they may have agreed with statements to portray themselves or their organization more favorably.

To summarize, the implementation of the CCM appeared to be functioning at the Sea Mar clinics and employees were relatively satisfied; $78 \%$ reported their job satisfaction was either excellent or very good. CCC's were valued within the Sea Mar system, especially with regard to patient self-management support, a key factor in improved patient outcomes for people with type 2 diabetes. CCC's were connecting with patients on a personal level, offering patients a person in whom they could confide and help them knit together and navigate the complex web of care needed for patients with chronic illnesses such as type 2 diabetes.

The fact that physicians and medical assistants represented the largest response groups may be due, in part, to the fact they have the greatest amount of contact with patients with diabetes and so were more likely to respond to the survey or, perhaps, they may have had the strongest opinions. Additionally, despite their short tenure, staff and providers employed for less than 1 year chose to respond to the survey, so they may have had strong feelings about the role or model and should continue to be included in such surveys.

\section{Acknowledgments}

This work was supported in part by grants from the Sinegal Foundation, and the National Institute of Health NCRR grant UL1RR025014.

\section{Disclosures}

The Sinegal Foundation and the National Institute of Health had no role in this research in terms of the formulation of the research questions, study design, data collection, and analysis and or decision to publish. There is no financial relationship between any of the authors who participated in this study and the Sinegal Foundation or the National Institute of Health.

\section{References}

1. Committee on Quality of Health Care in America Institute of Medicine. Crossing the quality chasm: a new health system for the 21 st century executive summary [report on the Internet]. Mar 2001 [cited June 14, 2012]; [about 6 p]. http://www.nap.edu/openbook.php?record_ id $=10027 \&$ page $=1$. Accessed June 14, 2012.

2. Centers for Disease Control and Prevention CDC.org [homepage on the Internet]. Chronic diseases: the power to prevent: at a glance 2009. Atlanta, GA [updated December 17, 2009; cited June 14, 2012]. http:// www.cdc.gov/chronicdisease/resources/publications/AAG/chronic.htm. Accessed June 14, 2012.

3. Partnership for Solutions: Johns Hopkins University, Baltimore for the Robert Wood Johnson Foundation Chronic conditions: making the case for ongoing care [report on the Internet] Sep 2004 [cited June 15, 2012]. http://www.rwjf.org/pr/product.jsp?id=14685. Accessed June 15, 2012. 
4. Bodenheimer T, Chen E, Bennett HD. Confronting the growing burden of chronic disease: Can the US health care workforce do the job? Health Aff (Millwood). 2009;28(1):64-74.

5. US Department of Health and Human Services. Office of Disease Prevention and Health Promotion. Healthy people 2020. [report on the Internet] Washington, DC. Mar 2010 [cited June 15, 2012]. http:// healthypeople.gov/2020/about/default.aspx. Accessed June 16, 2012.

6. Centers for Disease Control and Prevention. CDC.org [homepage on the Internet] National diabetes fact sheet 2011. Atlanta, GA. [cited June 14, 2012]. http://www.cdc.gov/diabetes/pubs/pdf/ndfs_2011.pdf. Accessed June 14, 2012.

7. Huang ES, Zhang Q, Brown SE, Drum ML, Meltzer DO, Chin MH. The cost-effectiveness of improving diabetes care in US federally qualified community health centers. Health Serv Res. 2007;42(6 Pt 1):2174-2193; discussion 2294-2323.

8. American Diabetes Association ADA.org [homepage on the Internet]. Direct and indirect costs of diabetes in the United States 2011 Phoenix AZ [cited June 14, 2012]. http://www.diabetesarchive.net/diabetesstatistics/cost-of-diabetes-in-us.jsp. Accessed June 14, 2012.

9. American Diabetes Association ADA.org [homepage on the Internet]. Total prevalence of diabetes and pre-diabetes. 2007 [cited June 20, 2012]. http://www.diabetesarchive.net/diabetes-statistics/prevalence. jsp. Accessed June 20, 2012.

10. Coleman K, Austin BT, Brach C, Wagner EH. Evidence on the Chronic Care Model in the new millennium. Health Aff (Millwood). 2009;28(1): 75-85.

11. Wagner EH, Bennett SM, Austin BT, Greene SM, Schaefer JK, VonkorffM. Finding common ground: patient-centeredness and evidence-based chronic illness care. J Altern Complement Med. 2005;11 Suppl 1: S7-S15.
12. Bodenheimer $\mathrm{T}$, Wagner $\mathrm{EH}, \mathrm{Grumbach} \mathrm{K}$. Improving primary care for patients with chronic illness. JAMA. 2002;288(14):1775-1779.

13. Bodenheimer $\mathrm{T}$, Wagner $\mathrm{EH}$, Grumbach $\mathrm{K}$. Improving primary care for patient with chronic illness: the chronic care model, part 2. JAMA. 2002;288(15):1909-1914.

14. Epping-Jordan J, Pruitt S, Bengoa R, Wagner EH. Improving the quality of health care for chronic conditions. Qual Saf Health Care. 2004;13(4):299-305.

15. Krause DS. Economic effectiveness of disease management programs: a meta-analysis. Dis Manag. 2005;8(2):114-134.

16. Wagner EH. Quality improvement can't be optional. Eff Clin Pract. 2001;4(6):278-280.

17. Siminerio LM, Piatt G, Zgibor JC. Implementing the chronic care model for improvements in diabetes care and education in a rural primary care practice. Diabetes Educ. 2005;31(2):225-234.

18. Hroscikoski MC, Solberg LI, Sperl-Hillen JM, Harper PG, McGrail MP, Crabtree BF. Challenges of change: a qualitative study of chronic care model implementation. Ann Fam Med. 2006;4(4):317-326.

19. Grembowski D. The Practice of Health Program Evaluation. Sherman Oaks: Sage; 2001

20. Bonomi AE, Wagner EH, Glasgow RE, VonKorff M. Assessment of chronic illness care (ACIC): a practical tool to measure quality improvement. Health Serv Res. 2002;37(3):791-820.
Journal of Multidisciplinary Healthcare

\section{Publish your work in this journal}

The Journal of Multidisciplinary Healthcare is an international, peerreviewed open-access journal that aims to represent and publish research in healthcare areas delivered by practitioners of different disciplines. This includes studies and reviews conducted by multidisciplinary teams as well as research which evaluates the results or conduct of such teams or

\section{Dovepress}

healthcare processes in general. The journal covers a wide range of areas and welcomes submission from practitioners at all levels, from all over the world. The manuscript management system is completely online and includes a very quick and fair peer-review system. Visit http://www.dovepress.com/testimonials.php to read real quotes from published authors. 